\title{
Human Dopamine Receptor-Conjugated Multidimensional Conducting Polymer Nanofiber Membrane for Dopamine Detection
}

\author{
Seon Joo Park, ${ }^{\dagger, \downarrow} \|$ Seung Hwan Lee, ${ }^{\S}$ Heehong Yang, ${ }^{\S}$ Chul Soon Park, " Chang-Soo Lee,
} Oh Seok Kwon, ${ }^{*}, \|$ Tai Hyun Park, ${ }^{*},, \perp$ and Jyongsik Jang ${ }^{*},+, \S$

${ }^{\dagger}$ Center for Integrated Smart Sensors (CISS), KAIST, Daejon 305-701, Republic of Korea
${ }^{\ddagger}$ World Class University Program of Chemical Convergence for Energy \& Environment (C2E2) and ${ }^{\S}$ School of Chemical and
Biological Engineering, Seoul National University (SNU), Seoul 151-744, Republic of Korea
${ }^{\|}$Harzards Monitoring BioNano Research Center, Korea Research Institute of Bioscience and Biotechnology (KRIBB), Daejeon
305-600, Republic of Korea
${ }^{\perp}$ Advanced Institutes of Convergence Technology, Suwon 443-270, Republic of Korea

Supporting Information

ABSTRACT: In the brain and central nervous system, dopamine plays a crucial role as a neurotransmitter or a local chemical messenger for interneuronal communication. Dopamine is associated with renal, hormonal, and cardiovascular systems. Additionally, dopamine dysfunction is known to cause serious illnesses, such as Parkinson's disease and Alzheimer's disease. Therefore, dopamine detection is essential for medical diagnosis and disease prevention and requires a novel strategy with high sensitivity and selectivity and a rapid response. Herein, we present a novel human dopamine receptor (hDRD1)-conjugated multidimensional conducting polymer nanofiber (NF) membrane for

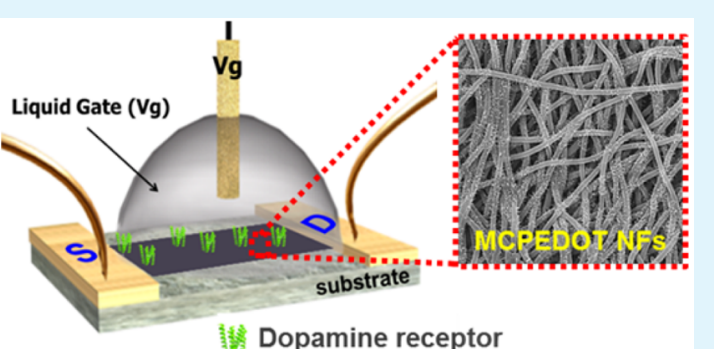

in Dopamine receptor the selective and sensitive detection of dopamine. The membrane, which consists of multidimensional carboxylated poly (3,4ethylenedioxythiophene) (MCPEDOT) NFs with nanorods, is used as a transistor in a liquid-ion gated field-effect transistor (FET)-based biosensor. Interestingly, hDRD1 is first expressed in Escherichia coli before it is immobilized onto the MCPEDOT NF. The hDRD1-MCPEDOT NF-based FET exhibits a rapid real-time response $(<2 \mathrm{~s})$ with high dopamine selectivity and sensitivity performance (approximately $100 \mathrm{fM}$ ). Furthermore, this FET device can be integrated into a poly(dimethylsiloxane)based microfluidic system and also can retain its high performance in the integrated system, which results in the generation of large-scale dopamine biosensors with a novel geometry.

KEYWORDS: multidimensional nanostructures, conducting polymers, human dopamine receptor, dopamine sensor, protein-based biosensor, microfluidic FET system

\section{INTRODUCTION}

Since the discovery of dopamine (DA) in the late 1950s, its function has been studied in a variety of systems. ${ }^{1} \mathrm{DA}$ is able to affect brain function and the central nervous system in its capacity as a catecholamine neurotransmitter. It also influences renal, hormonal, and cardiovascular systems. ${ }^{2,3}$ DA has been implicated in several diseases, including Parkinson's disease, schizophrenia, and Huntington's disease, and DA detection is therefore essential for early diagnosis and treatment of these diseases. ${ }^{1,4,5}$ Various detection techniques have been developed, such as high-performance liquid chromatography (HPLC), mass spectroscopy, and spectrophotometry. ${ }^{6-9}$ However, although these techniques can detect DA, they are limited by their high cost, low sensitivity, and variable label response. Electrochemical sensors based on organic and inorganic electrodes have also been introduced for DA analysis, but they showed low sensitivity. ${ }^{10-19}$ In our previous work, we designed an hDRD1-containing nanovesicle for DA detection. However, this also had limitations such as low sensitivity and slow response time because the nanovesicle-based biosensor operated through an indirect DA detection mechanism via calcium influx. ${ }^{20}$ Therefore, a new generation of DA detectors must be developed.

Recently, field-effect transistor (FET)-based biosensors have gained attention due to their low cost, easy operation, fast response, label-free operation, and high sensitivity. ${ }^{21-23}$ However, further enhancements are required to obtain a biosensor geometry that provides high performance and exhibits improved sensitivity and selectivity. ${ }^{20}$ For example, although Lin et al. demonstrated highly sensitive biosensors based on polysilicon nanowire FET, the selectivity would have a limitation because of chemical probe. ${ }^{23}$ To achieve these goals, several factors should be considered, these include increasing the surface area for enhanced interactions and using gate-

Received: August 19, 2016

Accepted: October 7, 2016

Published: October 7, 2016 
potential modulators to enable selective responses. ${ }^{20,24}$ To increase the surface area, several nanomaterials have been developed, including one-dimensional (1D) conducting polymer (CP) nanomaterials with unique electrical and physical properties. $^{25-29}$ Interestingly, manipulating the morphology of $\mathrm{CP}$ nanomaterials, particularly multidimensional $\mathrm{CP}$ nanomaterials, has increased the surface area and improved the functionality of these nanomaterials. ${ }^{30,31} \mathrm{We}$ previously developed a multidimensional CP nanomaterial-based toxic gas sensor system, and we demonstrated that increasing the surface area improved the sensing performance. ${ }^{24,32}$ Therefore, using multidimensional CP nanomaterials in biosensing systems, especially for DA detection, may provide highperformance sensing.

In addition, we have emphasized the importance of using a gate modulator for highly selective and rapid real-time response. $^{20}$ We previously demonstrated an application of high-performance FET systems by combining conductive nanomaterials as the signal transducers and $G$ protein-coupled receptors (GPCRs) as the recognition elements. ${ }^{33-35}$ GPCRs play a central role in the physiological functions of mammalian organisms in hormonal, neuronal, and sensory signaling processes. $^{36-38}$ We successfully achieved the overexpression of GPCRs in a bacterial expression system, ${ }^{39,40}$ which has the advantage of simplicity, convenience, low cost, and high productivity. ${ }^{40}$ In addition, GPCRs interact with their counterpart molecules in a highly selective and sensitive manner, providing substantial benefits as gate modulators in recognition systems. $^{41-43}$ The DA receptor, a member of the GPCR superfamily, selectively binds to DA in mixtures. ${ }^{36,37}$ Therefore, the combination of DA receptors and multidimensional $\mathrm{CP}$ nanostructures is an ideal approach for improving the FET system for DA detection, and it can provide information to support DA-related medical diagnosis and disease prevention.

In this study, we report a novel liquid ion-gated FET system with a dopamine receptor-conjugated multidimensional $\mathrm{CP}$ nanofiber membrane that exhibits a selective and sensitive response to DA. A multidimensional carboxylated poly $(3,4-$ ethylenedioxythiophene) (MCPEDOT) nanofiber (NF) with a nanorod (NR) membrane was fabricated and integrated as a transducer into a liquid-ion gated FET system. Human dopamine receptor (hDRD1: DR) was successfully expressed in Escherichia coli and purified, and its amine groups were chemically immobilized to the carboxyl groups of the MCPEDOT NF membrane. The DR-MCPEDOT NF in the FET system demonstrated excellent real-time responses with high selectivity and sensitivity for DA detection. The minimum detectable level (MDL) for DA was approximately $100 \mathrm{fM}$, and this sensitivity is approximately 2 times greater than that our previous biosensor for DA detection. Our biological application of multidimensional CP nanofibers will improve the performance of biosensing systems.

\section{EXPERIMENTAL SECTION}

Materials. 3,4-Ethylenedioxythiophene (EDOT), 2,3-dihydrothieno(3,4-b)[1,4]dioxine-2-carboxylic acid (COOH-EDOT), poly(methyl methacrylate) (PMMA $M_{\mathrm{w}}=350000$, Aldrich), dimethylformamide (DMF), and 4-(4,6-dimethoxy-1,3,5-triazin-2-yl)-4-methylmorpholinium chloride (DMT-MM) were obtained from SigmaAldrich.

Preparation of the PMMA NF Template. First, PMMA was dissolved in DMF for $12 \mathrm{~h}$ at $70-80{ }^{\circ} \mathrm{C}$. The PMMA NFs were fabricated from the PMMA/DMF solution by an electrospinning process. Specifically, the electrospun PMMA NFs were fabricated through a needle, which is made of stainless steel, with 22 gauge supplied by a portable dc power source (Nano NC $60 \mathrm{kV} / 2 \mathrm{~mA}$ ). Continuous injection was conducted by a syringe pump ( $\mathrm{Kd}$ Scientific) at a rate of $10 \mu \mathrm{m} \mathrm{min}{ }^{-1}$. To collect PMMA NFs on a cellulose substrate, the stainless needle was supplied with a high voltage output of $12 \mathrm{kV}$ and connected to a grounded collector that was $15 \mathrm{~cm}$ away from the needle. ${ }^{24}$

Fabrication of the Pristine Carboxylated Poly(3,4-ethylenedioxythiophene) (CPEDOT) NFs and MCPEDOT NFs on the PMMA NF Template. To fabricate multidimensional conducting polymer nanofibers, first, a ferric chloride, as an initiator, $\left(\mathrm{FeCl}_{3}\right.$, Aldrich)/methanol solution $(40 \mathrm{~mL})$ was absorbed onto the surface of the resulting PMMA NFs. After vacuum drying, the nanofibers with the adsorbed ferric chloride were transferred to a homemade pressurecontrolled glass chamber. Subsequently, liquid monomers (EDOT or an EDOT/carboxylated EDOT ([EDOT]/[COOH-EDOT] $=4 / 1$ ) mixture) were inserted under a controlled temperature and pressure, which resulted in the fabrication of pristine CPEDOT NFs surfaces (at $90^{\circ} \mathrm{C}$ and 1 Torr) and MCPEDOT NFs with nanorods (at $60{ }^{\circ} \mathrm{C}$ and 760 Torr) after $4 \mathrm{~h}^{30,44}$ The final dimensions of the MCPEDOT NFs and CPEDOT NFs membranes were approximately $12 \mathrm{~mm} \times 12 \mathrm{~mm}$ $\times 25 \mu \mathrm{m}$ (in length, width, and height).

Covalent DR Attachment on the Surface of NFs Grown on the Membrane. A similar procedure for covalently binding of the DR to the nanomaterials was introduced in our previous report. ${ }^{27,45}$ Specifically, the microelectrode glass substrate was modified with amine groups $\left(-\mathrm{NH}_{2}\right)$ by an overnight treatment with $10 \mathrm{wt} \%$ (3aminopropyl)trimethoxysilane solution. The carboxylic groups $(-\mathrm{COOH})$ of surface of the MCPEDOT NFs were reacted with the amine groups of the microelectrode substrate using a condensing reagent, 1 wt \% 4-(4,6-dimethoxy-1,3,5-trizin-2-yl)-4-methylmorpholinium chloride (DMT-MM) aqueous solution, for $12 \mathrm{~h}$. Continuously, the carboxylic groups on the opposite side of the membranes were covalently connected to the amine groups of DR by $1 \mathrm{wt} \%$ DMT-MM solution for $6 \mathrm{~h}$, which resulted in strong amide covalent bonding (-CONH-) between the DR and MCPEDOT NFs.

Fabrication of the Electrodes. A positive photoresist (AZ5214, Clariant Corp.) was coated onto a glass wafer by spin-coater, and patterns were formed via ultraviolet (UV) exposure through a highresolution film mask, followed by image reversal and development. The source and drain electrodes $(\mathrm{Cr} / \mathrm{Au}, 20 \mathrm{~nm} / 200 \mathrm{~nm}$, respectively) were deposited by thermal evaporation followed by a lift-off process.

Fabrication of the Poly(dimethylsiloxane) (PDMS)-Based Microfluidic Channel. The microchannel was fabricated using a standard soft lithography method and replica molding techniques. ${ }^{42}$ First, a negative photoresist (SU-8 2075, MicroChem Corp.) was coated onto a silicon wafer using a spin-coater and then soft baked. The microchannel pattern was formed via UV exposure through a high-resolution film mask. A master mold was prepared using postexposure baking and development. PDMS (Sylgard 184, DowCorning) and a curing agent were mixed according to a fixed ratio $(10: 1)$. A degassing process was progressed to remove air bubbles from the PDMS mixture. The silicon master was inserted into the mixture and baked in an oven. The cured PDMS mixture was peeled from the master mold, and inlet and outlet reservoirs were formed by punching holes into the PDMS. The PDMS was cleaned using oxygen plasma and bonded to the glass containing the source and drain electrodes and the DR MCPEDOT NF membrane.

Sensing Tests. The real-time response was obtained from electrical signals that were measured with a Keithley 2612A SourceMeter connected to a probe station (MS-TECH, MODEL $4000)$. The current change was normalized according to the equation $\Delta I / I_{0}=\left(I-I_{0}\right) / I_{0}$, where $I_{0}$ and $I$ indicate the initial and measured real-time currents, respectively.

Cloning the hDRD1 Gene. The hDRD1 genes (GenBank accession No. NC000005.9) were amplified by polymerase chain reaction (PCR). The amplified PCR product was inserted into pENTR/D-TOPO (Invitrogen). Then, the hDRD1 genes of pENTR/ 
D-TOPO were inserted into a pDEST15 vector (Invitrogen) using an LR Clonase-based recombination reaction.

Preparation of the HDRD1 Protein Using E. coli. The constructed pDEST15/GST-tag/hDRD1 vector was transformed into E. coli BL21(DE3). The hDRD1 expression was induced by adding $0.5 \mathrm{mM}$ isopropyl $\beta$-D-thiogalactoside (IPTG). After the cells were collected by centrifugation at $4{ }^{\circ} \mathrm{C}$ and $7000 \mathrm{~g}$ for $20 \mathrm{~min}$, the cells were disrupted by sonication. To collect the insoluble fraction containing cell debris and inclusion bodies, the centrifugation process was performed with the above-mentioned conditions. The desired fraction was resuspended in PBS and treated with 5\% Triton X-100 to solubilize the impurities. The fraction was then recollected after centrifugation $\left(4^{\circ} \mathrm{C}, 7000 \mathrm{~g}, 20 \mathrm{~min}\right)$, resuspended in PBS, and stored at $-80{ }^{\circ} \mathrm{C}$. The hDRD1 expression was analyzed by Western blot analysis using mouse anti-GST (Santa Cruz Biotechnology).

Fluorescence Images. DRs were labeled with Alexa Fluor488 sulfodichlorophenol esters (Alexa Fluor488-5-SDP ester, Invitrogen) according to the manufacture's protocol. The DRs $(2 \mathrm{mg} / \mathrm{mL})$ were mixed with the Alexa Fluor488-5-SDP ester $(0.25 \mathrm{mg})$ for labeling reaction of amine groups in PBS buffer containing $0.1 \mathrm{M}$ sodium bicarbonate. Then, labeled DRs were attached MCPEDOT NFs using 1 wt \% DMT-MM solution for $12 \mathrm{~h}$. After MCPEDOT NFs were washed with PBS to remove unreacted labeling reagent and DRs, fluorescence signal was monitored using a microscope (TE300, Nikon).

\section{RESULTS AND DISCUSSION}

Previously, we synthesized multidimensional nanostructures with poly(3,4-ethylenedioxythiophene) (PEDOT) as transistors for chemiresistive sensor applications. ${ }^{24,30,32}$ The MCPEDOT NFs with a NR membrane were fabricated using vapor deposition polymerization (VDP) and transferred to the FET substrate. The FET substrate was functionalized by the addition of carboxyl groups using a mixture of the carboxylated EDOT monomer and a regular EDOT monomer, and it was stored in the glass container and maintained at a controlled temperature and atmospheric pressure. The resulting MCPEDOT NF membranes were fabricated through VDP on the surface of the template PMMA NFs. Figure 1 illustrates the method of fabricating MCPEDOT NF membranes and the field-emission scanning electron microscopy (FE-SEM) images of both the
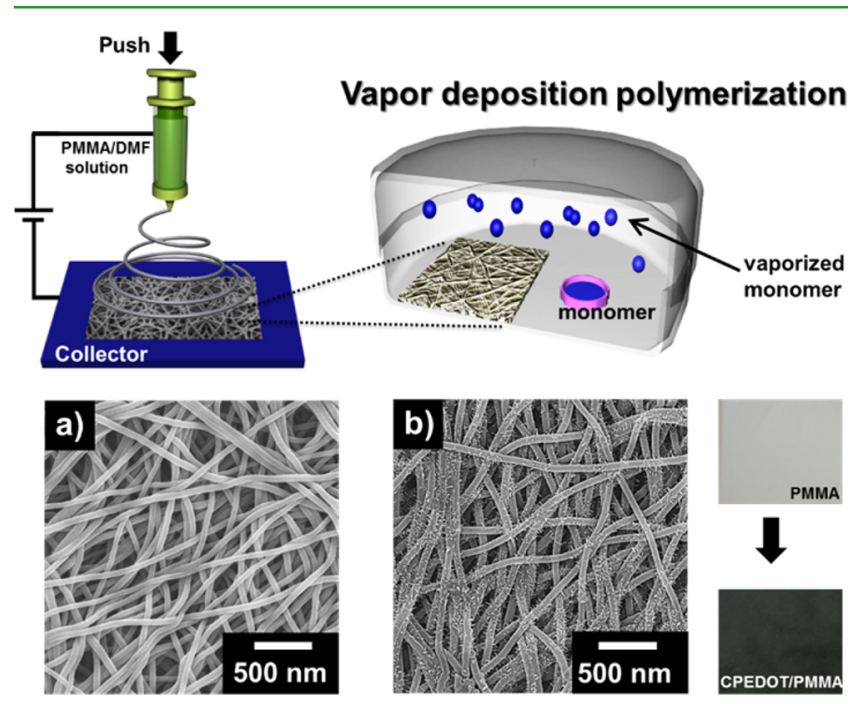

Figure 1. Schematic illustration of the fabrication of the MCPEDOT NF membrane by electrospinning and vapor deposition polymerization. FE-SEM images of (a) the PMMA template and (b) the MCPEDOT NF membrane.
PMMA template and the membrane, which showed that the PMMA diameter was approximately $60 \mathrm{~nm}$ (Figure 1a), and the shell thickness increased after the formation of MCPEDOT on the PMMA template (Figure 1b, MCPEDOT NFs are approximately $100 \mathrm{~nm}$ in diameter). Moreover, the color of the membrane was changed after VDP: the upper image on the right side of Figure 1 represents the PMMA template, and the image below represents the CPEDOT/PMMA nanofiber membrane.

The MCPEDOT NFs were successfully transferred on the glass electrodes to demonstrate the FET-based biosensor geometry. Interestingly, the NRs were grown on the surface of the smooth CPEDOT NFs, which resulted in a larger surface area than that of conventional ID nanostructures (Figure S1). The DR was expressed in E. coli using methods similar to those used to produce other GPCRs, as described previously. ${ }^{20,26,38}$ The molecular weight of the DR was confirmed using Western blot analysis with an anti-GST antibody (Figure 2a). The DR was covalently attached to the surface of the MCPEDOT NFs by a chemical reaction between the carboxyl $(-\mathrm{COOH})$ group of MCPEDOT NFs and the amine $\left(-\mathrm{NH}_{2}\right)$ group of the DR. Figures $2 \mathrm{~b}$ and $2 \mathrm{c}$ are typical FE-SEM images of the FET sensor platform of MCPEDOT NFs (b) without and (c) with DR. The energy-dispersive X-ray spectroscopy (EDS) were also observed to confirm chemical compositions of MCPEDOT NFs and smooth CPEDOT NFs (Table S1). Both of membranes showed similar chemical compositions after conducting polymer deposition. Moreover, the DR attachment on the MCPEDOT NFs was characterized by fluorescence images, as shown Figures $2 \mathrm{~d}$ and 2e. The FE-SEM analysis indicated that the surface of the MCPEDOT NFs became rougher after the introduction of the DR. Immobilizing the DR onto the surface is advantageous due to the physical stability of the DR in the liquid phase and its efficient electron pathway for the FET transistor.

To evaluate the electrical properties associated with the geometry of this DR-MCPEDOT NF sensor, we measured the current-voltage $(I-V)$ curves of the DR-MCPEDOT NFs on the electrodes. As shown in Figure 3a, linear $I-V$ curves were obtained over a range of -0.5 and $+0.5 \mathrm{~V}$ before and after the immobilization of DRs on the surface of MCPEDOT NFs, which indicates that a highly stable ohmic contact was preserved after introduction of the DRs. These results suggest that the transducer with the DR-MCPEDOT NF membrane could provide a reliable electrical signal due to detection of the analytes. To investigate the possibility of transducing the signal with the DR-MCPEDOT NFs, the liquid-ion gated FET system was designed using the DR-MCPEDOT NFs as the channels. PBS ( $\mathrm{pH}$ 7.4) was used as the electrolyte to enable the efficient control of the gate (Figure $3 \mathrm{~b}$ ). Generally, the environmental stability of the transducer is a highly important factor in the construction of biosensing systems because of the liquid state of the analytes. The significant binding event between the transducer and target analytes occurs in a liquid environment; thus, high-performance biosensors require stable electrical properties in the liquid state. Therefore, in this study, we introduced the FET system, which consists of a liquid-ion gate with PBS, for real-time DA detection. Liquid-ion gates provides two primary advantages for biosensors: first, they enable the formation of a substantial contact area on the surface of the DR-MCPEDOT NFs through a fixed gating electrode, and second, they enable amplifying the current of the binding responses from the biosensors. Figure $3 c$ displays the output 

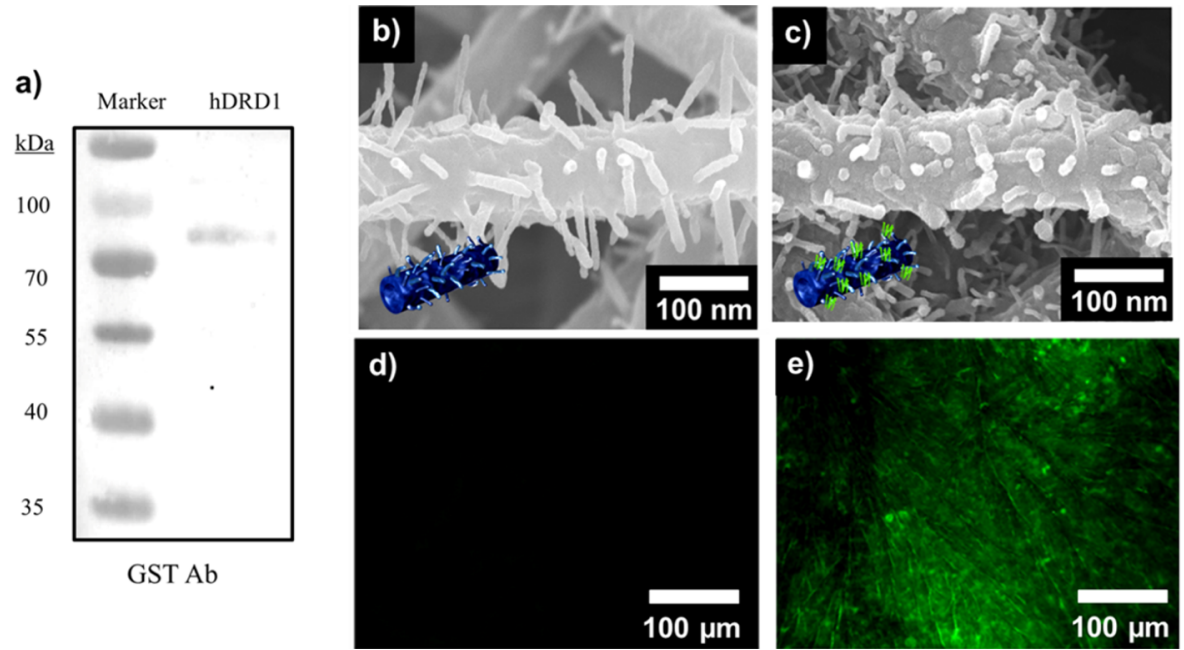

Figure 2. (a) Western blot analysis of purified DR (hDRD1) using an anti-GST antibody. Typical FE-SEM images of MCPEDOT membranes: (b) pristine MCPEDOT NFs and (c) dopamine-receptor conjugated MCPEDOT NFs. The insets are schematic illustrations of MCPEDOT NFs with and without dopamine receptors. Fluorescence images of (d) MCPEDOT NF membrane and (e) MCPEDOT NF membrane with Alexa Fluor488labeled DR.

a)

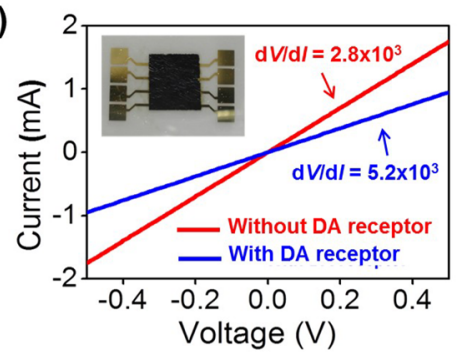

b)

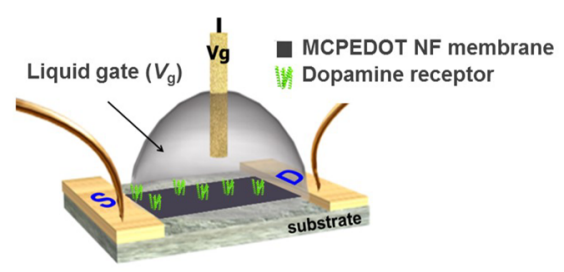

c)

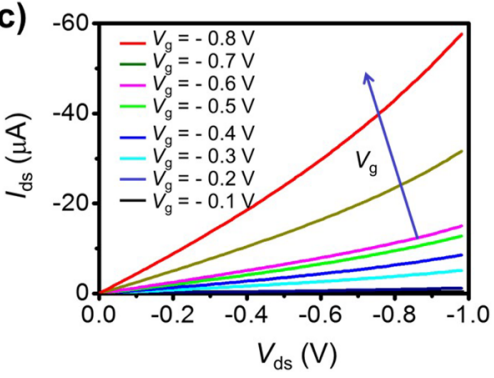

Figure 3. (a) Current-voltage $(I-V)$ curves of MCPDOT NFs on the glass electrode before (red line) and after (blue line) the introduction of DRs ( $V$ scan rate $\left.=10 \mathrm{mV} \mathrm{s}^{-1}\right)$. (b) Schematic diagram of a liquidion gated FET dopamine biosensor using DR MCPEDOT NF membrane $\left(V_{\mathrm{g}}\right.$ : gate voltage; $\mathrm{S}$ : source electrode; and $\mathrm{D}$ : drain electrode). (c) $I_{\mathrm{ds}}-V_{\mathrm{ds}}$ output characteristics of DR-MCPEDOT NF membrane FET geometry at different $V_{\mathrm{g}}$ from -0.1 to $-0.8 \mathrm{~V}$ in steps of $-0.1 \mathrm{~V}\left(V_{\mathrm{ds}}: 0\right.$ to $-0.5 \mathrm{~V}$ in a step of $\left.-50 \mathrm{mV}\right)$.

characteristics of the liquid-ion gated FET device with the DRMCPEDOT NF membrane at ambient temperature. The negatively increasing gate voltages lead to an increasing drainto-source current $\left(I_{\mathrm{ds}}\right)$ because a negative charge applied on the surface of the DR-MCPEDOT NF changes the state of the intrinsic oxidation level in the $\mathrm{CP}$ chains. The results in Figure $3 c$ show that the liquid-ion gated FET system with DRMCPEDOT NF has a clearly defined p-type transistor characteristic with holes as the primary charge carrier. Therefore, we can monitor the binding events at different DA concentrations by measuring real-time changes in the current of the DR-MCPEDOT NF FET biosensor under controlled gate voltages.

In previous works, ${ }^{24,32,46}$ important parameters, including the thickness, types of nanomaterials on the surface, nanomaterial density, and alignment of the NFs, were used to design highperformance FET biosensors with membrane NFs. In this work, the effect of the surface dimension of the MCPEDOT $\mathrm{NF}$ membrane was investigated because the capacity of the surface for DR attachment is the most important factor that determines the performance. Figure $4 \mathrm{a}$ shows the real-time responses of liquid-ion gated FETs fabricated using DAMCPEDOT NF membranes with different surface dimensions. The sensitivity increased as the surface area of the membrane increased because of the increased surface capacity for DR attachment. ${ }^{24}$ In further experiments, an MCPEDOT NF membrane with dimensions of $12 \mathrm{~mm} \times 12 \mathrm{~mm} \times 25 \mu \mathrm{m}$ (length, width, and height, respectively) was fixed because the sample loading size of the homemade electrodes had a maximum width of $12 \mathrm{~mm}$.

The FET-based DA biosensor showed a highly rapid, sensitive, and selective real-time response toward DA. To demonstrate the sensing capability of the DR-MCPEDOT NFbased FET-type DA biosensor in a liquid environment (PBS), the dependence of the change in $I_{\mathrm{ds}}$ the DA concentrations was monitored at fixed values of $V_{\mathrm{ds}}=-50 \mathrm{mV}$ and $V_{\mathrm{g}}=-50 \mathrm{mV}$. The DRs immobilized on the surface of the MCPEDOT NF selectively bind DA, which induces electrical changes due to the rearrangement of DR on the MCPEDOT NFs substrate. The interactions between DA and the DRs can indirectly affect the charge carrier density on the surface of the MCPEDOT NFs. The real-time responses from the FET DA biosensors was continuously monitored while the DA concentration was increased (Figure 4b). In the two different FET-based biosensors based on DR-MCPEDOT NF and DR-CPEDOT 

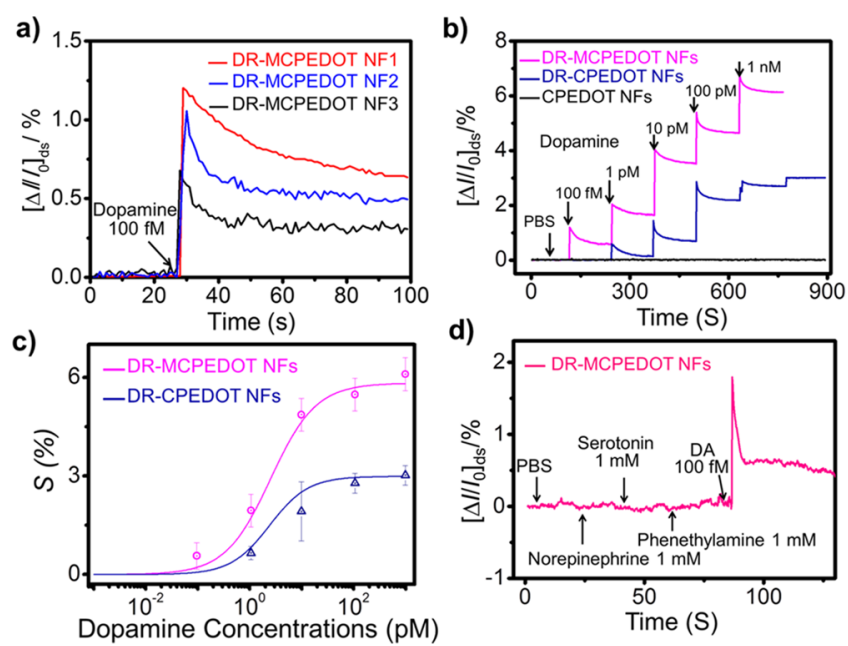

Figure 4. (a) Real-time responses of DR-MCPEDOT NF1, NF2, and NF3 with various surface dimensions (length, width, and height, respectively: $12 \mathrm{~mm} \times 12 \mathrm{~mm} \times 25 \mu \mathrm{m}$, MCPEDOT NF1; $12 \mathrm{~mm} \times$ $8 \mathrm{~mm} \times 25 \mu \mathrm{m}$; MCPEDOT NF2; and $12 \mathrm{~mm} \times 4 \mathrm{~mm} \times 25 \mu \mathrm{m}$; MCPEDOT NF3) for dopamine concentration of $100 \mathrm{fM}$. (b) Sensing performance of liquid ion-gated FET dopamine biosensors with DRMCPEDOT NFs, DR-CPEDOT NFs, and CPEDOT NFs for dopamine concentrations from $100 \mathrm{fM}$ to $1 \mathrm{nM}$ at $V_{\mathrm{ds}}=-10 \mathrm{mV}$ $\left(\Delta I / I_{0}=\left(I-I_{0}\right) / I_{0}\right.$, where $I_{0}$ is the initial current and $I$ is the instantaneous current). (c) Calibration curves of the dopamine biosensors from (b). (d) DA selectivity of DR-MCPEDOT NFs.

NF membranes, $I_{\mathrm{ds}}$ increased as the DA concentrations increased. This is caused by the accumulation of hole-type charge carriers during the DR-DA rearrangement. Specifically, the negative charges near the DR-MCPEDOT NF surface are generated by the specific binding event between DR and DA, resulting in the indirect gating effect for the MCPEDOT NF transistor. Consequently, the hole-charge carriers accumulate as the DA concentration increases, which results in the modulation of the current. In addition, the MDL of DRMCPEDOT NFs was 10 times higher than that of the DRCPEDOT because of the larger surface areas. In the control experiment, no significant signals were obtained from pristine CPEDOT NFs using this sensing mechanism. This DRMCPEDOT NF FET biosensor provides excellent sensing performance with respect to its sensitivity, as demonstrated by an MDL of approximately $100 \mathrm{fM}$, which is an increase of nearly 2 orders of magnitude compared to that of the previous DA biosensing system (our previous biosensor was a nanovesicle-containing hDRD1-based FET system, which was indirectly controlled by calcium influx). ${ }^{20}$ More interestingly, the response time of the FET-based DA biosensor to DA was less than $1 \mathrm{~s}$ because the signaling transfer time of DR in a neuron is less than $1 \mathrm{~s}$. The stability time and recovery time were ca. $25 \mathrm{~s}$ and ca. $50 \mathrm{~s}$, respectively.

To determine the relationship between the DA sensing property and the multidimensional nanostructure with an increased surface areas, the dose-response curves were characterized using the normalized values of the current change values, as shown in Figure 4c. Compared with the DRCPEDOT NFs, the DR-MCPEDOT NFs have higher halfmaximal responses (EC50 at $2.47 \mathrm{pM}$ ) to DA (Figure $4 \mathrm{~b}$ ). Moreover, the equilibrium constant $K$ can be calculated by the Langmuir isotherm model: $K_{\mathrm{DR}-\mathrm{MCPEDOT} \mathrm{NFs}}\left(4.474 \times 10^{14} \mathrm{M}^{-1}\right)$ was higher than $K_{\text {DR-CPEDOT NFs }}\left(1.127 \times 10^{14} \mathrm{M}^{-1}\right)$, which indicates greater sensitivity for DA detection. To evaluate the selectivity of the biosensors, control samples were prepared with neurotransmitters and precursors. In Figure 4d, the FETtype DA biosensor shows a highly selective response for control samples with similar structures. There were no significant changes in $I_{\mathrm{ds}}$ when $1 \mathrm{mM}$ norepinephrine, $1 \mathrm{mM}$ serotonin, and $1 \mathrm{mM}$ phenethylamine were injected, while a current $\left(I_{\mathrm{ds}}\right)$ change was observed when the $100 \mathrm{fM}$ DA sample was injected. Based on those results, the DR-MCPEDOT NF membrane can be used as a high-performance DA biosensor.

Microfluidic systems have numerous advantages, such as low sample consumption and reagent cost, and they facilitate precise analysis through automatic control of the flow through the system. Therefore, these system have been used for a wide range of applications, including the detection of chemical species. ${ }^{8,42}$ To exploit the above advantages, a DR-MCPEDOT NF membrane-based FET system was integrated with a PDMSbased microfluidic system (Figure 5a,b and Figure S2). First, a
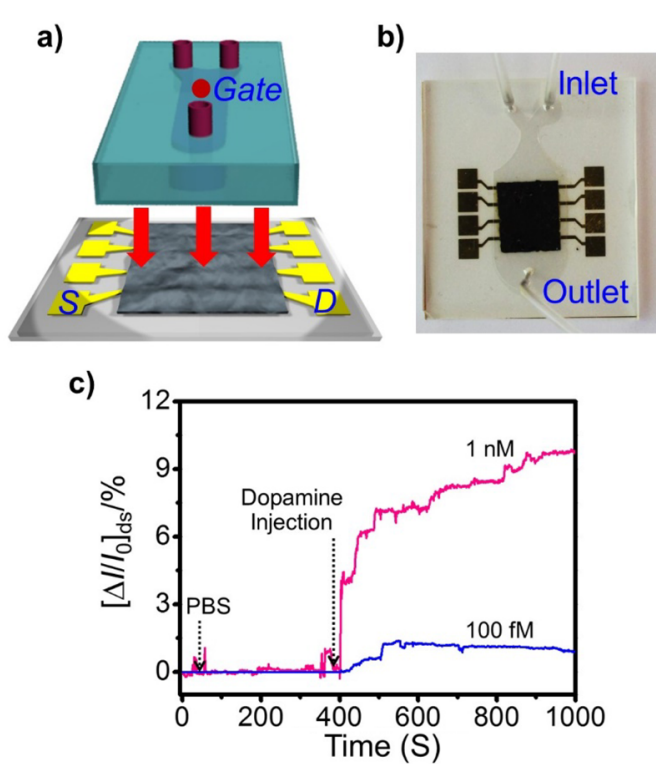

Figure 5. (a) Schematic illustration of the fluidic FET-type DA biosensor with DR-MCPEDOT NFs. (b) Photography of the microfluidic system with the integrated of FET geometry and PDMS microchannel. (c) $I_{\mathrm{ds}}$ responses of the DR-MCPEDOT NFs in the microfluidic system for the dopamine concentrations range from $100 \mathrm{fM}$ to $1 \mathrm{nM}\left(V_{\mathrm{ds}}=-10 \mathrm{mV}\right)$.

PBS buffer solution was used to obtain a baseline flow rate of 1 $\mu \mathrm{L} \mathrm{min}{ }^{-1}$. A constant source-drain bias of $V_{\mathrm{ds}}=-10 \mathrm{mV}$ and a constant gate bias of $V_{\mathrm{g}}=0 \mathrm{~V}$ were applied until the initial current required to obtain the baseline was stabilized. Next, 100 $\mathrm{fM}$ and $1 \mathrm{nM}$ DA solutions were injected at a flow rate of $1 \mu \mathrm{L}$ $\mathrm{min}^{-1}$. Figure $5 \mathrm{c}$ shows the change in the current of the DRMCPEDOT NF membrane in the fluidic system at the fixed values of $V_{\mathrm{g}}$ and $V_{\mathrm{ds}}$. As the DA solution was injected into the microfluidic system, the current gradually increased. Furthermore, a larger change in current was observed when a highconcentration DA solution was injected into the microchannel. These results indicate that solution leakage did not occur in the system, and they further show that the sensing behavior of the DR-MCPEDOT NF membrane in the microfluidic system is similar to that of the DR-MCPEDOT NF membrane surrounded by a stagnant electrolyte. 


\section{CONCLUSION}

In summary, we demonstrated that a DR-conjugated multidimensional conducting polymer nanofiber membrane can be used as a biosensor to detect dopamine molecules. The DR was successfully expressed in E. coli and integrated into the MCPEDOT NF membrane-based FET system by covalent bonding. This system exhibited a real-time response to DA along with high sensitivity and selectivity: the MDL of the system was approximately $100 \mathrm{fM}$ for DA detection, which indicates that this level of sensitivity can be achieved by the combination of DRs and the MCPEDOT NF membrane. The MCPEDOT NF membrane provide a large surface area for functionalization and enhanced the sensing performance, which is based on the selectivity and sensitivity of DR. The system can be used as a portable, inexpensive tool for medical diagnosis and the evaluation of drug efficacy.

\section{ASSOCIATED CONTENT}

\section{S Supporting Information}

The Supporting Information is available free of charge on the ACS Publications website at DOI: 10.1021/acsami.6b10437.

Additional information about SEM and TEM images, PDMS-based microfluidic preparation process, and chemical compositions (PDF)

\section{AUTHOR INFORMATION}

\section{Corresponding Authors}

*(O.S.K.) Tel +82-42-879-8462; Fax +82-42-879-8462; e-mail oskwon79@kribb.re.kr.

*(T.H.P.) Tel +82-2-880-8020; Fax +82-2-875-9348; e-mail thpark@snu.ac.kr.

*(J.J.) Tel +82-2-880-7069; Fax +82-2-888-1604; e-mail jsjang@plaza.snu.ac.kr.

\section{Author Contributions}

S.J.P. and L.S.H. contributed equally to this work.

\section{Notes}

The authors declare no competing financial interest.

\section{ACKNOWLEDGMENTS}

This research was supported by the National Research Foundation funded by the Korean Government (MSIP, No. 2015065103) and the Brain Research Program of National Research Foundation of Korea (NRF), which is funded by the Ministry of Science, ICT \& Future Planning (NRF2016M3C7A1905384) and the KRIBB Initiative Research Program. This work was supported by Development Fund of Seoul National University funded by Dongjin Semichem. Co., Korea (0458-20130066).

\section{REFERENCES}

(1) Wightman, R. M.; May, L. J.; Michael, A. C. Detection of Dopamine Dynamics in the Brain. Anal. Chem. 1988, 60, 769A-793A.

(2) Cooper, J. R.; Bloom, F. E.; Roth, R. H. The Biochemical Basis of Neuropharmacology, 8th ed.; Oxford University Press: New York, 2003.

(3) Jin, G.; Zhang, Y.; Cheng, W. Poly (P-Aminobenzene Sulfonic Acid)-Modified Glassy Carbon Electrode for Simultaneous Detection of Dopamine and Ascorbic Acid. Sens. Actuators, B 2005, 107, 528534.

(4) Savitt, J. M.; Dawson, V. L.; Dawson, T. M. Diagnosis and Treatment of Parkinson Disease: Molecules to Medicine. J. Clin. Invest. 2006, 116, 1744-1754.
(5) Seeman, P.; Niznik, H. B.; Guan, H.; Booth, G.; Ulpian, C. Link between D1 and D2 Dopamine Receptors Is Reduced in Schizophrenia and Huntington Diseased Brain. Proc. Natl. Acad. Sci. U. S. A. 1989, 86, 10156-10160.

(6) Chan, E.; Wee, P.; Ho, P.; Ho, P. High-Performance Liquid Chromatographic Assay for Catecholamines and Metanephrines Using Fluorometric Detection with Pre-Column 9-Fluorenylmethyloxycarbonyl Chloride Derivatization. J. Chromatogr., Biomed. Appl. 2000, 749, 179-189.

(7) Hows, M. E. P.; Lacroix, L.; Heidbreder, C.; Organ, A. J.; Shah, A. J. High-Performance Liquid Chromatography/Tandem Mass Spectrometric Assay for the Simultaneous Measurement of Dopamine, Norepinephrine, 5-Hydroxytryptamine and Cocaine in Biological Samples. J. Neurosci. Methods 2004, 138, 123-132.

(8) Lee, S. H.; Lim, J. H.; Park, J.; Hong, S.; Park, T. H. Bioelectronic Nose Combined with a Microfluidic System for the Detection of Gaseous Trimethylamine. Biosens. Bioelectron. 2015, 71, 179-185.

(9) Nezhad, M. H.; Tashkhourian, J.; Khodaveisi, J. Sensitive Spectrophotometric Detection of Dopamine, Levodopa and Adrenaline Using Surface Plasmon Resonance Band of Silver Nanoparticles. $J$. Iran. Chem. Soc. 2010, 7, S83-S91.

(10) Fabre, B.; Taillebois, L. Poly (Aniline Boronic Acid)-Based Conductimetric Sensor of Dopamine. Chem. Commun. 2003, 24, 2982-2983.

(11) Gao, F.; Cai, X.; Wang, X.; Gao, C.; Liu, S.; Gao, F.; Wang, Q. Highly Sensitive and Selective Detection of Dopamine in the Presence of Ascorbic Acid at Graphene Oxide Modified Electrode. Sens. Actuators, B 2013, 186, 380-387.

(12) Gao, X. P.; Zheng, G.; Lieber, C. M. Subthreshold Regime Has the Optimal Sensitivity for Nanowire Fet Biosensors. Nano Lett. 2009, $10,547-552$.

(13) Goyal, R. N.; Gupta, V. K.; Bachheti, N.; Sharma, R. A Electrochemical Sensor for the Determination of Dopamine in Presence of High Concentration of Ascorbic Acid Using a FullereneC60 Coated Gold Electrode. Electroanalysis 2008, 20, 757-764.

(14) Mao, Y.; Bao, Y.; Gan, S.; Li, F.; Niu, L. Electrochemical Sensor for Dopamine Based on a Novel Graphene-Molecular Imprinted Polymers Composite Recognition Element. Biosens. Bioelectron. 2011, 28, 291-297.

(15) Min, K.; Yoo, Y. J. Amperometric Detection of Dopamine Based on Tyrosinase-Swnts-Ppy Composite Electrode. Talanta 2009, 80, 1007-1011.

(16) Sun, C.-L.; Chang, C.-T.; Lee, H.-H.; Zhou, J.; Wang, J.; Sham, T.-K.; Pong, W.-F. Microwave-Assisted Synthesis of a Core-Shell Mwcnt/Gonr Heterostructure for the Electrochemical Detection of Ascorbic Acid, Dopamine, and Uric Acid. ACS Nano 2011, 5, 77887795.

(17) Tang, H.; Lin, P.; Chan, H. L.; Yan, F. Highly Sensitive Dopamine Biosensors Based on Organic Electrochemical Transistors. Biosens. Bioelectron. 2011, 26, 4559-4563.

(18) Zhang, H.-M.; Li, N.-Q.; Zhu, Z. Electrocatalytic Response of Dopamine at a Dl-Homocysteine Self-Assembled Gold Electrode. Microchem. J. 2000, 64, 277-282.

(19) Zhao, H.; Zhang, Y.; Yuan, Z. Study on the Electrochemical Behavior of Dopamine with Poly (Sulfosalicylic Acid) Modified Glassy Carbon Electrode. Anal. Chim. Acta 2001, 441, 117-122.

(20) Park, S. J.; Song, H. S.; Kwon, O. S.; Chung, J. H.; Lee, S. H.; An, J. H.; Ahn, S. R.; Lee, J. E.; Yoon, H.; Park, T. H.; Jang, J. Human Dopamine Receptor Nanovesicles for Gate-Potential Modulators in High-Performance Field-Effect Transistor Biosensors. Sci. Rep. 2014, 4, 4342 .

(21) Li, B.-R.; Chen, C.-W.; Yang, W.-L.; Lin, T.-Y.; Pan, C.-Y.; Chen, Y.-T. Biomolecular Recognition with a Sensitivity-Enhanced Nanowire Transistor Biosensor. Biosens. Bioelectron. 2013, 45, 252259.

(22) Li, B.-R.; Hsieh, Y.-J.; Chen, Y.-X.; Chung, Y.-T.; Pan, C.-Y.; Chen, Y.-T. An Ultrasensitive Nanowire-Transistor Biosensor for Detecting Dopamine Release from Living PC12 Cells under Hypoxic Stimulation. J. Am. Chem. Soc. 2013, 135, 16034-16037. 
(23) Lin, C.-H.; Hsiao, C.-Y.; Hung, C.-H.; Lo, Y.-R.; Lee, C.-C.; Su, C.-J.; Lin, H.-C.; Ko, F.-H.; Huang, T.-Y.; Yang, Y.-S. Ultrasensitive Detection of Dopamine Using a Polysilicon Nanowire Field-Effect Transistor. Chem. Commun. 2008, 44, 5749-5751.

(24) Kwon, O. S.; Park, S. J.; Lee, J. S.; Park, E.; Kim, T.; Park, H.-W.; You, S. A.; Yoon, H.; Jang, J. Multidimensional Conducting Polymer Nanotubes for Ultrasensitive Chemical Nerve Agent Sensing. Nano Lett. 2012, 12, 2797-2802.

(25) Kwon, O. S.; Ahn, S. R.; Park, S. J.; Song, H. S.; Lee, S. H.; Lee, J. S.; Hong, J.-Y.; Lee, J. S.; You, S. A.; Yoon, H. Ultrasensitive and Selective Recognition of Peptide Hormone Using Close-Packed Arrays of Hpthr-Conjugated Polymer Nanoparticles. ACS Nano 2012, 6, $5549-5558$.

(26) Lee, S. H.; Kwon, O. S.; Song, H. S.; Park, S. J.; Sung, J. H.; Jang, J.; Park, T. H. Mimicking the Human Smell Sensing Mechanism with an Artificial Nose Platform. Biomaterials 2012, 33, 1722-1729.

(27) Song, H. S.; Kwon, O. S.; Lee, S. H.; Park, S. J.; Kim, U.-K.; Jang, J.; Park, T. H. Human Taste Receptor-Functionalized Field Effect Transistor as a Human-Like Nanobioelectronic Tongue. Nano Lett. 2012, 13, 172-178.

(28) Yoon, H.; Lee, S. H.; Kwon, O. S.; Song, H. S.; Oh, E. H.; Park, T. H.; Jang, J. Polypyrrole Nanotubes Conjugated with Human Olfactory Receptors: High-Performance Transducers for Fet-Type Bioelectronic Noses. Angew. Chem., Int. Ed. 2009, 48, 2755-2758.

(29) Yoon, H. Current Trends in Sensors Based on Conducting Polymer Nanomaterials. Nanomaterials 2013, 3, 524-549.

(30) Kwon, O. S.; Park, S. J.; Park, H.-W.; Kim, T.; Kang, M.; Jang, J.; Yoon, H. Kinetically Controlled Formation of Multidimensional Poly (3, 4-Ethylenedioxythiophene) Nanostructures in Vapor-Deposition Polymerization. Chem. Mater. 2012, 24, 4088-4092.

(31) Nguyen, D. N.; Yoon, H. Recent Advances in Nanostructured Conducting Polymers: From Synthesis to Practical Applications. Polymers 2016, 8, 118.

(32) Kwon, O. S.; Park, S. J. Highly Sensitive and Selective Chemiresistive Sensors Based on Multidimensional Polypyrrole Nanotubes. Chem. Commun. 2012, 48, 10526-10528.

(33) Kim, T. H.; Lee, S. H.; Lee, J.; Song, H. S.; Oh, E. H.; Park, T. H.; Hong, S. Single-Carbon-Atomic-Resolution Detection of Odorant Molecules Using a Human Olfactory Receptor-Based Bioelectronic Nose. Adv. Mater. 2009, 21, 91-94.

(34) Kim, T. H.; Song, H. S.; Jin, H. J.; Lee, S. H.; Namgung, S.; Kim, U.-k.; Park, T. H.; Hong, S. Bioelectronic Super-Taster” Device Based on Taste Receptor-Carbon Nanotube Hybrid Structures. Lab Chip 2011, 11, 2262-2267.

(35) Song, H. S.; Jin, H. J.; Ahn, S. R.; Kim, D.; Lee, S. H.; Kim, U.K.; Simons, C. T.; Hong, S.; Park, T. H. Bioelectronic Tongue Using Heterodimeric Human Taste Receptor for the Discrimination of Sweeteners with Human-Like Performance. ACS Nano 2014, 8, 97819789.

(36) Beck-Sickinger, A. G.; Budisa, N. Genetically Encoded Photocrosslinkers as Molecular Probes to Study G-Protein-Coupled Receptors (GPCRs). Angew. Chem., Int. Ed. 2012, 51, 310-312.

(37) Rasmussen, S. G.; Choi, H.-J.; Rosenbaum, D. M.; Kobilka, T. S.; Thian, F. S.; Edwards, P. C.; Burghammer, M.; Ratnala, V. R.; Sanishvili, R.; Fischetti, R. F. Crystal Structure of the Human \&Bgr; 2 Adrenergic G-Protein-Coupled Receptor. Nature 2007, 450, 383-387.

(38) Rosenbaum, D. M.; Rasmussen, S. G.; Kobilka, B. K. The Structure and Function of G-Protein-Coupled Receptors. Nature 2009, 459, 356-363.

(39) Song, H. S.; Lee, S. H.; Oh, E. H.; Park, T. H. Expression, Solubilization and Purification of a Human Olfactory Receptor from Escherichia Coli. Curr. Microbiol. 2009, 59, 309-314.

(40) Yang, H.; Song, H. S.; Ahn, S. R.; Park, T. H. Purification and Functional Reconstitution of Human Olfactory Receptor Expressed in Escherichia Coli. Biotechnol. Bioprocess Eng. 2015, 20, 423-430.

(41) Kwon, O. S.; Song, H. S.; Park, S. J.; Lee, S. H.; An, J. H.; Park, J. W.; Yang, H.; Yoon, H.; Bae, J.; Park, T. H. An Ultrasensitive, Selective, Multiplexed Superbioelectronic Nose That Mimics the Human Sense of Smell. Nano Lett. 2015, 15, 6559-6567.
(42) Lee, S. H.; Oh, E. H.; Park, T. H. Cell-Based Microfluidic Platform for Mimicking Human Olfactory System. Biosens. Bioelectron. 2015, 74, 554-561.

(43) Park, S. J.; Kwon, O. S.; Lee, S. H.; Song, H. S.; Park, T. H.; Jang, J. Ultrasensitive Flexible Graphene Based Field-Effect Transistor (Fet)-Type Bioelectronic Nose. Nano Lett. 2012, 12, 5082-5090.

(44) Lee, J. E.; Lee, Y.; Ahn, K.-J.; Huh, J.; Shim, H. W.; Sampath, G.; Im, W. B.; Huh, Y. I.; Yoon, H. Role of Co-Vapors in Vapor Deposition Polymerization. Sci. Rep. 2015, 5, 8420.

(45) Kwon, O. S.; Park, S. J.; Jang, J. A High-Performance Vegf Aptamer Functionalized Polypyrrole Nanotube Biosensor. Biomaterials 2010, 31, 4740-4747.

(46) Kwon, O. S.; Park, E.; Kweon, O. Y.; Park, S. J.; Jang, J. Novel Flexible Chemical Gas Sensor Based on Poly (3, 4-Ethylenedioxythiophene) Nanotube Membrane. Talanta 2010, 82, 1338-1343. 